\title{
Stochastic Filtering Technique for UAV-Based Communications On The Move Terminal Tracking Accuracy Evaluation
}

\author{
Saki Omi, Hyo-Sang Shin, Antonios Tsourdos \\ The School of Aerospace, Transport and Manufacturing \\ Cranfield University \\ Cranfield, U.K. \\ \{s.omi, h.shin, a.tsourdos\}@cranfield.ac.uk,
}

\author{
Joakim Espeland, Andrian Buchi \\ QuadSAT \\ Odense, Denmark \\ $\{j e, a b\} @$ quadsat.com
}

\begin{abstract}
Along with the growth of communication and satellite industry, the importance of satellite antenna evaluation is increasing. Particularly Communication On The Move (COTM) terminal antenna, including the communication between new types of constellations on LEO and MEO, requires tracking accuracy test for the communication on moving vehicles. The conventional test facilities are locally fixed and lack flexibility. To make the antenna measurement more accessible, we are developing a methodology for in-situ measurement by introducing multiple Unmanned-Aerial-Vehicles (UAVs) system with RF payload. Thanks to the dynamic flexibility of UAVs, this system can flexibly change the test configuration on site and make new test scenarios available, such as emulating the orbit of non-GEO satellites during the measurement. However, one of the challenges of the proposed system is the additional uncertainties during the measurement due to the mobility of UAVs. To overcome this challenge, we design recursive stochastic filtering and fusion approaches, and evaluate their estimation performance via numerical simulations. By introducing stochastic filter and fusion algorithms, the effect of error is mitigated, and better accuracy can be achieved compared to an existing method. This project is performed in collaboration with Cranfield University in the UK and QuadSAT in Denmark.
\end{abstract}

\section{INTRODUCTION}

Communication is one of the key technology nowadays and satellite communication is one of them. The number of the satellite in the orbit keep increasing and the number of the terrestrial terminal antennas is also growing. The communication between LEO and MEO satellites is also emerging. The satellite communication is getting more congested and this environment contains more risk of communication interference. To mitigate the risk to keep the secure communication, it is certainly beneficial to evaluate the terminal antennas and their system before and/or after their implementation. COTM is a communication when the terminal antenna is mounted on the moving vehicles such as a car, an airplane, a train and a ship. One of the important requirements of COTM terminal antenna is the tracking capability to keep pointing towards the intended satellite when it is operated on the vehicle. For the tracking evaluation test, it has been requiring a huge facility or involvement of the satellite in operation. Fraunhofer IIS in collaboration with Ilmenau University of
Technology has established Facility for Over-the-air Research and Testing (FORTE) which can evaluate the tracking accuracy [1]. This facility was authorized by a antenna testing entity of Global VSAT Forum (GVF) [2]. It can provide the terminal evaluation very precisely. However the facility is locally fixed and cannot adjust the location of the sensors during the measurement. These characteristics could limit the accessibility and flexibility of the antenna measurement and may not be efficient enough to measure all coming antennas in short time. To overcome these limitation and accelerate the measurement process in this current trend, we have been proposing insitu measurement using a multi-UAV system [3]. With this system, the antenna can be tested on-site by delivering the testing equipment and also it adds more flexibility for test scenarios, thanks to the mobility of the UAVs. For instance, it can emulate the trajectory of the non-GEO satellite during the measurement.

On the other hand, RF measurement by UAVs is attracting a lot of attention recently because of the improvement of RF and UAV technologies [4], [5]. However the focus of these works is on the creation of radiation pattern, and it is not targeting the tracking accuracy of COTM.

Depointing measurement is an evaluation which assumes that the radiation pattern of Antenna Under the Test (AUT) is known and can be used as a reference. In the existing approach, the depointing is estimated from the correlation between the known reference radiation pattern and the received signal strength during the measurement [1]. This is referred as table matching method in this work. However when the UAV system is involved in the measurement, additional uncertainties will be introduced. So, it would be beneficial to have a methodology to reduce the effect of noise and improve the accuracy of the estimation. Also, to keep the test setup as simple and low-cost as possible, it is preferable to keep the required number of the UAVs with RF sensors. To overcome these challenges, stochastic filtering approaches are formulated in a unique way in this work. 


\section{The Stochastic Filtering Technique}

\section{A. Overview of Kalman Filter}

To estimate the existing depointing angle, estimation theory is considered in this work. Estimation theory is a branch of statistics to deal with the estimation of the values of the parameters of interest based on measured data. The knowledge of the state of interest is described by probability density function (pdf) and it is described as $p(x)$ where $x$ is the parameter vector of interest. It can be possible to improve the estimation accuracy when some information related to the value of the parameters of interest. The available information could be the measurement and system models, measurement data and knowledge about initial condition. Given a measurement $z$, the state of knowledge can be changed (possibly improved) to conditional pdf as $p(x \mid z)$ as the posterior distribution.

Kalman filter is one of the most popular algorithms for state prediction in linear system. Given the process model which represents the propagation of the state from time $k-1$ to $k$, it recursively provides the statistically optimal estimate $\hat{x}_{k}$ which minimises $E\left[\left(x_{k}-\hat{x}_{k}\right)\left(x_{k}-\hat{x}_{k}\right)^{T}\right]$ where $x_{k}$ is the true value and $E[\cdot]$ denotes an expectation operator. The popularity of Kalman filter is based on the fact that it can incorporate measurement and modelling noise effects in a relatively simple form. Also, it includes the statistical measure of the uncertainty which allows to evaluate the system and each sensor's performance with error covariance matrix $P$.

\section{B. Kalman filter algorithm}

The dynamic model of the target is generally described as Linear Time Invariant (LTI) Model [6].

$$
X_{k+1}=F X_{k}+G u_{k}+\Gamma w_{k}
$$

where $F$ is the state transition matrix, $G$ is control-input matrix to the control vector $u_{k}, \Gamma$ is a noise matrix and $w_{k}$ is the process noise which is assumed to be zero-mean Gaussian with covariance $Q$, i.e. $w_{k} \sim \mathcal{N}(0, Q)$.

For the measurement model which describes the relationship between the current state and measurement follows;

$$
z_{k+1}=H X_{k+1}+v_{k+1}
$$

where $H$ is the measurement matrix and $v_{k+1}$ is the measurement noise vector which is also assumed to be zero-mean Gaussian with covariance $R$, i.e. $v_{k+1} \sim \mathcal{N}(0, R)$.

Kalman filter algorithm consists of two stages, which are prediction and correction, and is described as below. Also, Fig. 1 visualises the procedure.

Prediction (model update):

$$
\begin{gathered}
\hat{X}_{k+1 \mid k}=F \hat{X}_{k \mid k}+G u_{k} \\
P_{k+1 \mid k}=F P_{k \mid k} F^{T}+\Gamma Q \Gamma^{T}
\end{gathered}
$$

Correction (measurement update):

$$
\begin{gathered}
v_{k+1}=z_{k+1}-H \hat{x}_{k+1 \mid k} \\
K_{k+1}=P_{k+1 \mid k} H^{T}\left(H P_{k+1 \mid k} H^{T}+R_{k+1}\right)^{-1}
\end{gathered}
$$

$$
\begin{gathered}
\hat{x}_{k+1 \mid k+1}=\hat{x}_{k+1 \mid k}+K_{k+1} v_{k+1} \\
P_{k+1 \mid k+1}=\left(I-K_{k+1} H_{k+1}\right) P_{k+1 \mid k}
\end{gathered}
$$

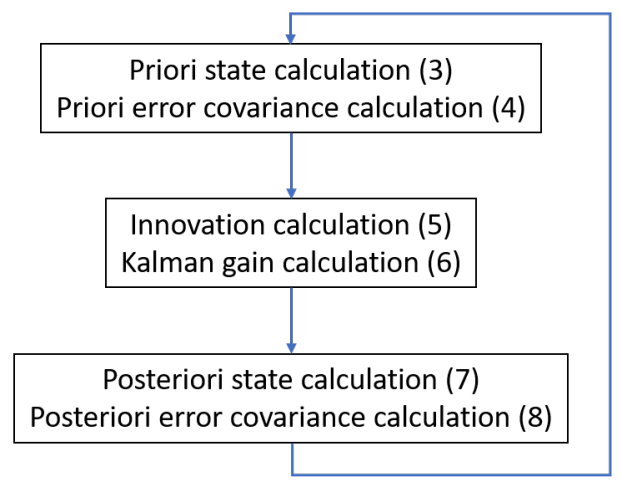

Fig. 1. Kalman Filter Procedure

\section{Extended Kalman Filter}

The assumption of linear state transition and linear measurement transition may not be applicable for some cases. To overcome this assumption and to deal with nonlinear models, Extended Kalman filter (EKF) can be used [7]. In EKF, the process and measurement model can be represented as

$$
\begin{gathered}
X_{k+1}=f\left(X_{k}, u_{k}\right)+\Gamma w_{k} \\
z_{k+1}=h\left(X_{k+1}\right)+v_{k+1}
\end{gathered}
$$

and $F_{k}$ and $H_{k}$ in Kalman filter algorithm can be obtained by linearization from first order Taylor expansion.

$$
\begin{aligned}
& \nabla f_{X}=\left.\frac{\partial f\left(X_{k}, u_{k}\right)}{\partial X_{k}}\right|_{X_{k}=X_{k+1 \mid k}} \\
& \nabla h_{X}=\left.\frac{\partial h\left(X_{k}, u_{k}\right)}{\partial X_{k}}\right|_{x_{k}=X_{k+1 \mid k}}
\end{aligned}
$$

Then, (3-8) are reformulated to Prediction (model update):

$$
\begin{gathered}
\hat{X}_{k+1 \mid k}=f\left(\hat{X}, u_{k}\right) \\
P_{k+1 \mid k}=\nabla f_{X} P_{k \mid k} \nabla f_{X}^{T}+\Gamma Q \Gamma^{T}
\end{gathered}
$$

Correction (measurement update):

$$
\begin{gathered}
v_{k+1}=z_{k+1}-h\left(\hat{X}_{k+1 \mid k}\right) \\
K_{k+1}=P_{k+1 \mid k} \nabla h_{X}^{T}\left(\nabla h_{X} P_{k+1 \mid k} \nabla h_{X}^{T}+R_{k+1}\right)^{-1} \\
\hat{X}_{k+1 \mid k+1}=\hat{X}_{k+1 \mid k}+K_{k+1} v_{k+1} \\
P_{k+1 \mid k+1}=\left(I-K_{k+1} \nabla h_{x}\right) P_{k+1 \mid k}
\end{gathered}
$$




\section{Multisensor Fusion}

Multisensor fusion techniques allow us to combine information and measurement data from different sources to identify a united picture [8]. The advantages of multisensor fusion are mainly the enhanced data authenticity and availability. It can improve the reliability and robustness, and increase the confidence. Also, it can extend the spacial and temporal coverage.

In this work, the three types of fusion architecture are tested. Measurement level fusion directly fuses the collected data from the sensors as illustrated by Fig. 2. The measurement data from each sensor are combined and the state of the target is estimated from the fused measurement data [9]. In this method, the dimension of the vector in the algorithm becomes larger. It can be realized by considering the matrices $z, H, R$ in (5)-(8) as

$$
\begin{gathered}
Z=\left[\begin{array}{c}
z_{1} \\
\vdots \\
z_{N}
\end{array}\right] \\
H=\left[\begin{array}{c}
H_{1} \\
\vdots \\
H_{N}
\end{array}\right] \\
R=\operatorname{diag}\left(R_{1}, \ldots, R_{N}\right) .
\end{gathered}
$$

State-vector fusion is another approach which uses a group of Kalman filters and local state estimations are obtained as shown in Fig. 3 . These estimated states are then centrally fused using a weighted sum of the state estimates [10]. The fusion algorithm for state and covariance matrix are described as below.

$$
\begin{gathered}
\hat{X}_{k \mid k}=\hat{X}_{1(k \mid k)}+P_{1(k \mid k)}\left[P_{1(k \mid k)}+P_{2(k \mid k)}\right]^{-1}\left[\hat{X}_{2(k \mid k)}-\hat{X}_{1(k \mid k)}\right] \\
P_{(k \mid k)}=P_{1(k \mid k)}-P_{1(k \mid k)}\left[P_{1(k \mid k)}+P_{2(k \mid k)}\right]^{-1} P_{1(k \mid k)}^{T}
\end{gathered}
$$

for sensor 1 and sensor 2. It is also possible to use the fused estimation for the prediction as illustrated in Fig.4. This method would improve the prediction with uncertain estimation but it requires more communication.

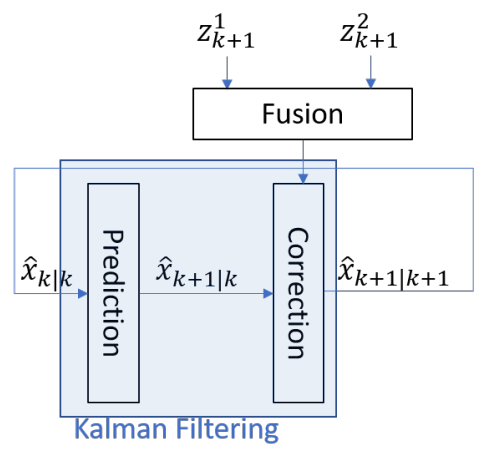

Fig. 2. Measurement level fusion

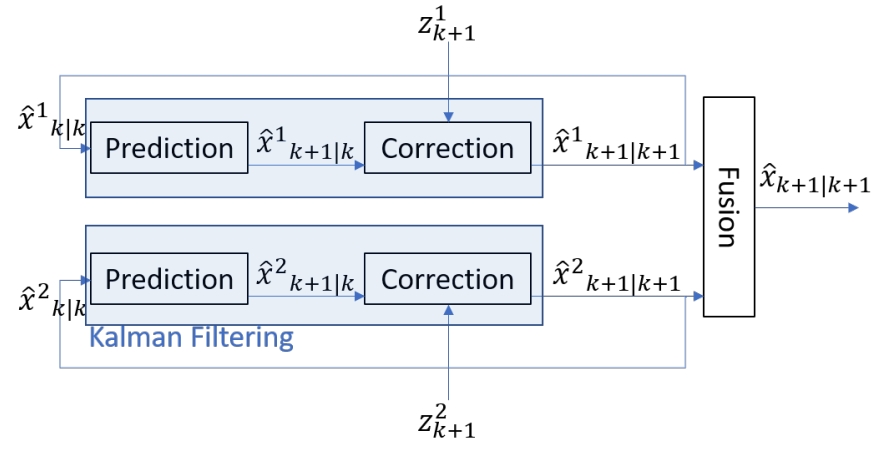

Fig. 3. State vector fusion 1

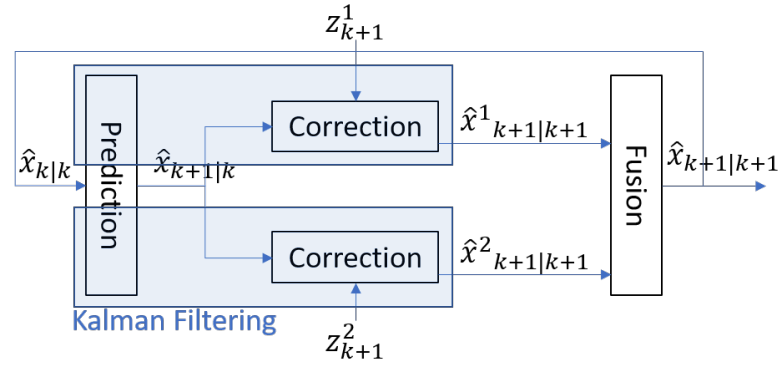

Fig. 4. State vector fusion2

\section{METHOD}

The contribution of this work is the uniqueness of the formulation of Kalman filter for depointing measurement. In this section, how it can be implemented is explained.

\section{A. Objective}

For the tracking accuracy evaluation, depointing angle $d \omega$ of Antenna under the Test (AUT) is the parameter of interest and it can be estimated by receiving signal from multiple UAVs (Fig. 5). Depointing measurement is the numerical angular measurement between target angle and the actual heading angle of the AUT. When the AUT is COTM terminal, it needs to keep compensating the motion of the vehicle and keep its line of sight (LOS) at the target satellite. Hence, the state to estimate is the heading angle in this evaluation test. In the considered test setup, the motion of the vehicle is emulated by the motion table underneath of the AUT during the measurement and RF receivers mounted on the UAVs are placed around the target LOS of the AUT.

\section{B. Process Model}

Target dynamics are represented as

$$
X_{k+1}=F X_{k}+G u_{k}+\Gamma w_{k}
$$

, where transformation matrix $F$ is identity matrix and state vector $X_{k}$ is

$$
X_{k}=\left[\begin{array}{l}
\omega_{a z_{A U T}} \\
\omega_{e l_{A U T}}
\end{array}\right],
$$




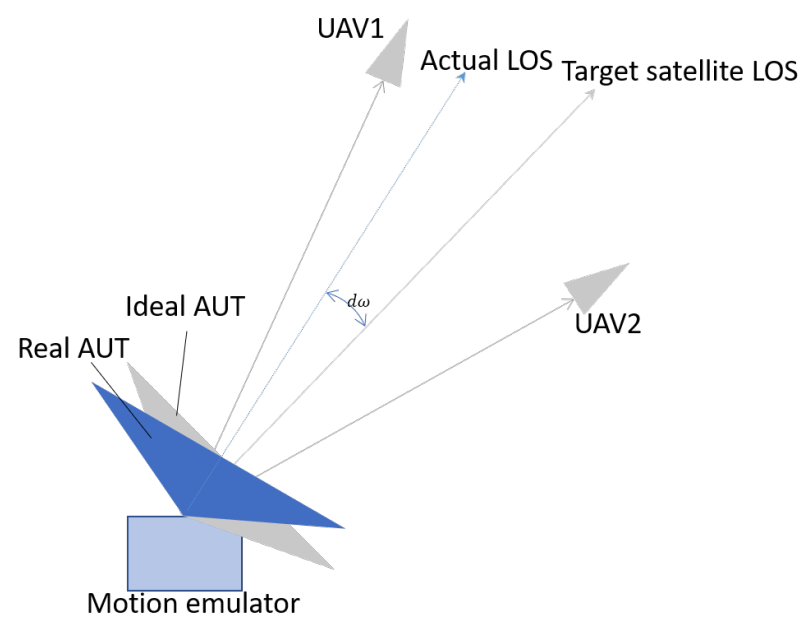

Fig. 5. Depointing measurement

$$
\begin{aligned}
G_{k} & =\left[\begin{array}{cc}
\Delta t & 0 \\
0 & \Delta t
\end{array}\right], \\
u_{k} & =\left[\begin{array}{l}
v_{a z}(k) \\
v_{e l}(k)
\end{array}\right] .
\end{aligned}
$$

$X_{k}$ is the state vector of the AUT which represent the heading angle in azimuth and elevation direction. $G u_{k}$ is implemented to consider the predefined target satellite velocity $\left(v_{a z}, v_{e l}\right)$ to follow the known target satellite trajectory. This is the vector representing the displacement of the target satellite during the time step $\Delta t$. Input matrix related to system noise $\Gamma$ is

$$
\Gamma=\left[\begin{array}{ll}
1 & 0 \\
0 & 1
\end{array}\right]
$$

and process noise coveriance $Q$ is defined as

$$
Q=\sigma_{w}^{2} .
$$

When the terminal antenna is operated with non-GEO satellite, the terminal antenna is required to follow the target satellite trajectory while they are also compensating the motion from the vehicle. When the state of the target is not stationary, the target dynamics model should consider angular velocity of the AUT. However because of the available measurement is limited to signal strength in this case, the state of the system including the velocity is unobservable. On the other hand, the satellite orbit is available information. Therefore, in this work, by assuming that the AUT has capability to approximately follow the defined target satellite trajectory including some error, the system can be formulated as (24).

\section{Sensor Observation Model}

To apply Kalman filter, the measurement equation needs to be described with respect to the parameter of interest. The observation $z_{k}$ is described with a non-linear function $h\left(X_{k+1}\right)$

$$
z_{k+1}=h\left(X_{k+1}\right)+v(k)
$$

Measurement noise coveriance matrix $R$ is

$$
R=\operatorname{diag}\left(\sigma_{v 1}^{2}, \ldots, \sigma_{v N}^{2}\right)
$$

where $N$ is the number of the sensors. Measurement equation $h(x)$ can be obtained as approximation based on the known radiation pattern in the form of $h\left(\omega_{a z}, \omega_{e l}\right)$. For this approximation, the method called spline interpolation was used.

The Jacobian of the observation model with respect to the targets states.

$$
\begin{gathered}
H_{k}=\left.\frac{\partial h}{\partial X}\right|_{X=X_{k \mid k-1}} \\
\frac{\partial h}{\partial X}=\left[\begin{array}{c}
-\left.\frac{\partial h}{\partial \omega_{a}}\right|_{\omega_{a z}=\omega_{a z_{U A V}}-\omega_{a z_{A U T}}} \\
-\left.\frac{\partial h}{\partial \omega_{e l}}\right|_{\omega_{e l}=\omega_{e l_{U A V}}-\omega_{e l_{A U T}}}
\end{array}\right]^{T}
\end{gathered}
$$

\section{Numerical Simulation}

The following simulation is considered to examine the performance of the Kalman filter based depointing estimation and compare the performance between conventional table matching approach.

In this experiment, random heading angular acceleration is added to the AUT model and generate the pseudo depointing angle. UAVs are positioned with some noise with respect to the LOS toward the intended satellite direction. Since the UAVs' positions are not mechanically fixed during the measurement, it would encounter some disturbances which could generate unwanted displacement of the sensors as illustrated as $d \psi, d \theta, d \phi$ and $d R$ in Fig. 6. When non-GEO scenario is used, the intended satellite direction becomes time variant and UAVs could have larger positioning error. In this simulation,

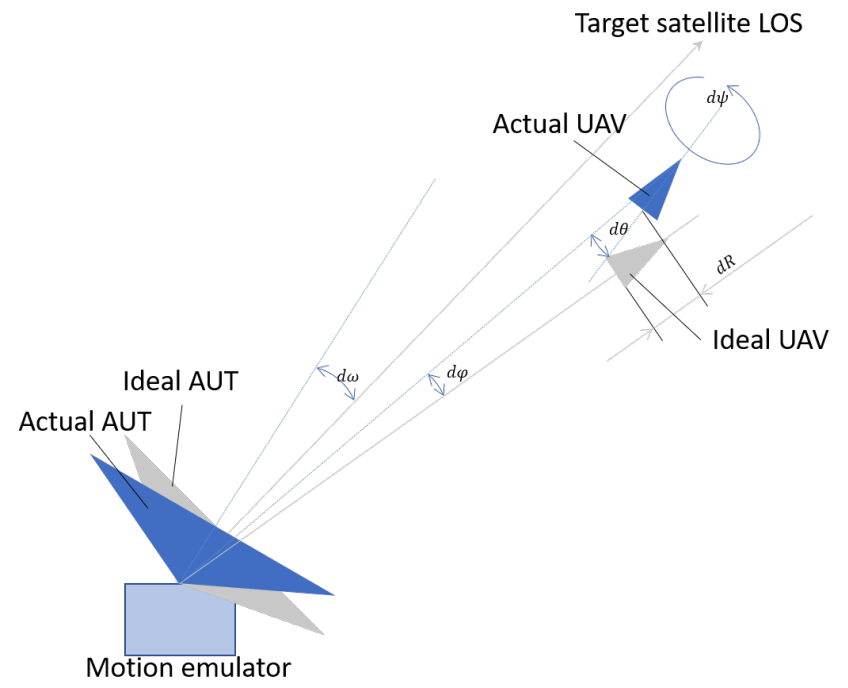

Fig. 6. Uncertainty of depointing measurement

these noise are taken into account as potential noise elements.

\section{A. Link Budget}

Based on Friis' transmission equation, the received power at each sensor is simulated as (34) in $d B$ [11]. 


$$
P_{r}=G_{r}+G_{t}+P_{t}+20 \log _{10} \frac{\lambda}{4 \pi R}-L_{p o l}
$$

where $L_{p o l}$ is loss coming from the polarization error and $L_{\text {loss }}$. By eliminating the constant values, the relative received signal power is

$$
P_{r}=G_{r}(\theta, d \theta)+G_{t}(\omega, d \omega, \psi, d \psi)-L
$$

where $L$ consists of polarization error loss and range error $(d R)$ loss;

$$
L=20 \log _{10} \frac{R+d R}{R}-20 \log _{10} \cos (d \phi)
$$

where $R$ is the target distance from the AUT. In the simulation, the random error for $d \psi, d \theta, d \phi$ and $d R$ are generated as Gaussian noise and random Gaussian noise is added to the simulated signal power based on the defined maximum possible Signal to Noise Ratio (SNR).

\section{B. Antenna Model}

The AUT model and the probe antenna mounted on the UAV are simulated to have 3.8 degree and 45 degree. To reduce the affect of $d \theta$, the probe antenna is selected to have a large beamwidth.

\section{Uncertainty}

Depointig measurement is numerically simulated in GEO and non-GEO scenario. It is assumed that the trajectory of the intended satellite is known and based on this information, the UAVs move as the time elapses to keep the formation around the LOS toward the target satellite. Because of the additional movement, non-GEO scenario contains more uncertainties in the positioning of the UAVs. These uncertainties are added as zero-mean normal distribution with the standard deviation in Table I

TABLE I

STANDARD DEVIATIONS FOR THE UAV POSITIONING UNCERTAINTY

\begin{tabular}{|c|c|c|c|c|}
\hline Scenario & $d R[\mathrm{~m}]$ & $d \theta[\mathrm{deg}]$ & $d \psi[\mathrm{deg}]$ & $d \omega[\mathrm{deg}]$ \\
\hline GEO & 0.01 & 0.01 & 2 & 1 \\
\hline Non-GEO & 0.02 & 0.02 & 4 & 2 \\
\hline
\end{tabular}

\section{Result AND ANALysis}

The performance of the Kalman filter based depointing estimations and table matching estimation are compared by changing the condition parameters such as the number of UAVs, SNR and the orbit of the target satellite.

Fig. 7 - Fig. 9 show the performance of each filters. The average error, after iterating the episode consists of 100 steps for 200 times, is shown by changing the number of the UAVs in the figures. EIF, SV, SV2 and TM represent the measurement level fusion, the first state-vector fusion, the second statevector fusion and table matching method respectively. Fig. 7 and Fig. 8 are for the scenario of GEO with SNR $40 \mathrm{~dB}$ and 50 dB. Fig. 9 is for non-GEO scenario. Also, Table II shows the numerical result about mean error and error standard deviation.
In any cases, Kalman filtering approach exceed the performance of table matching approach. When estimation is performed based on the measurement from a single UAV, the table matching approach has no capability to select a single estimation with reasoning behind. This is because all of the points which has the same reference value have equal amount of possibility to be the state of AUT. On the other hand, Kalman filter algorithm can take the sequential estimation into account and eliminate the fantom state estimation.

The performance of measurement level fusion and the second vector fusion shows the best accuracy and standard deviation and their performance does not change so much if more than 3 UAVs are involved. On the other hand, the table matching method showed more improvement when more sensors are available.

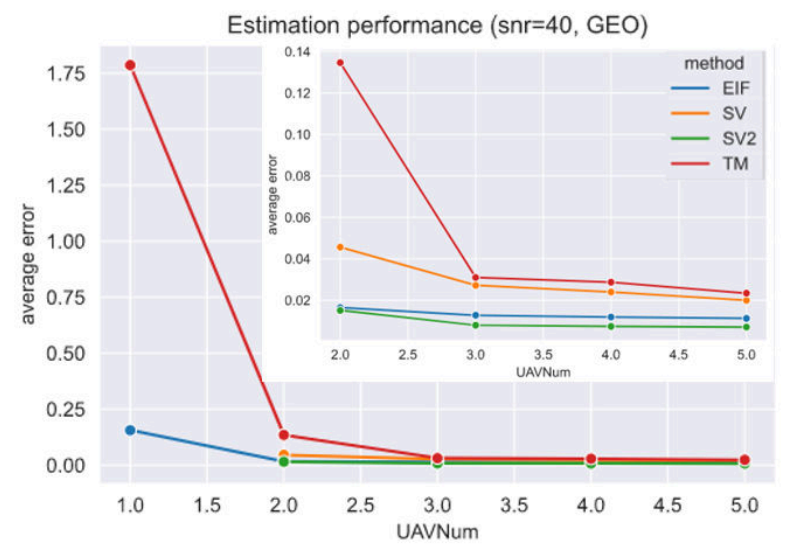

Fig. 7. $\mathrm{SNR}=40 \mathrm{~dB}, \mathrm{GEO}$

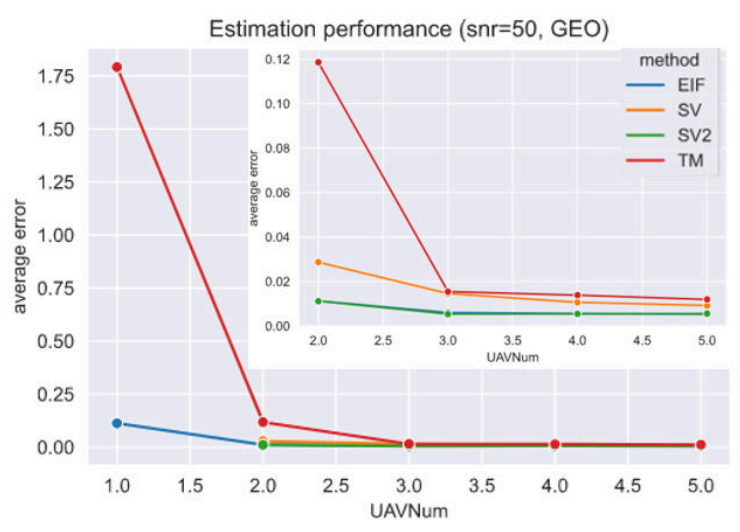

Fig. 8. $\mathrm{SNR}=50 \mathrm{~dB}, \mathrm{GEO}$

\section{CONCLUSION}

In this work, stochastic filter for depointing measurement was formulated and its performance was analyzed. The developed approach showed the better accuracy and it requires less number of the sensors in the numerical simulation compared to the conventional table matching approach. Considering the 


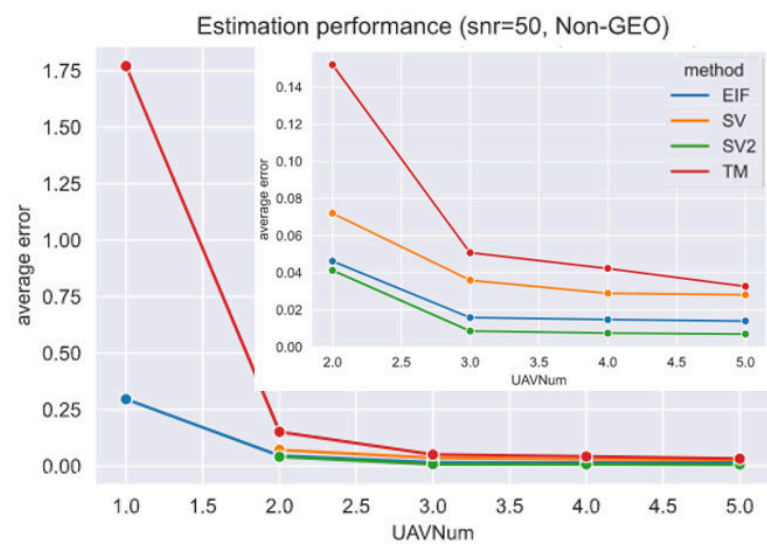

Fig. 9. $\mathrm{SNR}=50 \mathrm{~dB}$, Non-GEO

TABLE II

NUMERICAL RESULT OF NON-GEO, SNR=50DB EXAMPLE

\begin{tabular}{cccc}
\hline UAV Number & method & mean error & error covariance \\
\hline 1 & EKF & 0.2960 & 0.3124 \\
& TM & 1.7701 & 0.8980 \\
\hline \multirow{2}{*}{2} & ML & 0.0462 & 0.0492 \\
& SV1 & 0.0720 & 0.0878 \\
& SV2 & 0.0413 & 0.0441 \\
& TM & 0.1519 & 0.5341 \\
\hline \multirow{3}{*}{3} & ML & 0.0158 & 0.0148 \\
& SV1 & 0.0360 & 0.0477 \\
& SV2 & 0.0086 & 0.0078 \\
& TM & 0.0507 & 0.0477 \\
\hline \multirow{3}{*}{4} & ML & 0.0147 & 0.0137 \\
& SV1 & 0.0289 & 0.0331 \\
& SV2 & 0.0075 & 0.0067 \\
& TM & 0.0423 & 0.0403 \\
\hline \multirow{2}{*}{5} & ML & 0.0140 & 0.0128 \\
& SV1 & 0.0281 & 0.0289 \\
& SV2 & 0.0070 & 0.0064 \\
& TM & 0.0327 & 0.0307 \\
\hline
\end{tabular}

additional uncertainty due to the dynamic sensors on the UAVs and the benefit to keeping the system simple and low cost, the developed methodology would be suitable for this application. In the future work, it would be beneficial to work on the optimization of the positioning of the UAVs with this approach and review the approximation method of the radiation pattern method.

\section{ACKNOWLEDGEMENT}

This work is partially funded by QuadSAT. We would like to thank them for their support.

\section{REFERENCES}

[1] M. Alazab, M. Rieche, G. Del Galdo, W. Felber, F. Raschke, G. Siegert, and M. Landmann, "On-earth performance evaluation of SatCom on-themove (SOTM) terminals," Proceedings - IEEE Military Communications Conference MILCOM, pp. 634-640, 2013.

[2] G. V. Forum, "Global VSAT Forum PERFORMANCE AND TEST GUIDELINES FOR TYPE APPROVAL OF' COMMS ON THE MOVE ' MOBILE SATELLITE GVF-105 COMMUNICATIONS,' pp. 1-30.
[3] S. Omi, H.-S. Shin, A. Tsourdos, J. Espeland, and A. Buchi, "Introduction to UAV swarm utilization for communication on the move terminals tracking evaluation with reinforcement learning technique," in 2021 15th European Conference on Antennas and Propagation (EuCAP), pp. 1-5, IEEE, 32021

[4] T. Fritzel, H. J. Steiner, and R. Straus, "Advances in the Development of an Industrial UAV for Large-Scale Near-Field Antenna Measurements," 13th European Conference on Antennas and Propagation, EuCAP 2019 , no. EuCAP, pp. 1-3, 2019.

[5] M. Garcia-Fernandez, Y. Alvarez Lopez, A. Arboleya, B. GonzalezValdes, Y. Rodriguez-Vaqueiro, M. E. De Cos Gomez, and F. LasHeras Andres, "Antenna Diagnostics and Characterization Using Unmanned Aerial Vehicles," IEEE Access, vol. 5, pp. 23563-23575, 2017.

[6] P. S. Maybeck, "Stochastic models, estimation, and control VOLUME 1," tech. rep., 1979.

[7] S. Thrun, W. Burgard, and D. Fox, "PROBABILISTIC ROBOTICS," tech. rep., 2005.

[8] B. Khaleghi, A. Khamis, F. O. Karray, and S. N. Razavi, "Multisensor data fusion: A review of the state-of-the-art," Information Fusion, vol. 14, no. 1, pp. 28-44, 2013.

[9] Q. Gan and C. Harris, "Comparison of two measurement fusion methods for Kalman-filter-based multisensor data fusion," IEEE Transactions on Aerospace and Electronic Systems, vol. 37, no. 1, pp. 273-279, 2001.

[10] G. Shivanand, K. V. K. Reddy, and D. B. Prasad, "An innovative asynchronous, multi-rate, multi-sensor state vector fusion algorithm for air defence applications," in IFAC-PapersOnLine, vol. 49, pp. 337-342, Elsevier B.V., 2016.

[11] G. Maral and M. Bousquet, Satellite Communications Systems: Systems, Techniques and Technology. Wiley, 6th Edition, 2020. 
$2021-12-06$

\title{
Stochastic filtering technique for
}

UAV-based communications on the

move terminal tracking accuracy evaluation

\author{
Omi, Saki
}

IEEE

Omi S, Shin H-S, Tsourdos A, et al., (2021) Stochastic filtering technique for UAV-based communications on the move terminal tracking accuracy evaluation. In: 2021 Antenna Measurement Techniques Association Symposium (AMTA), 24-29 October 2021, Daytona Beach, USA

https://doi.org/10.23919/AMTA52830.2021.9620662

Downloaded from Cranfield Library Services E-Repository 